\title{
In vitro susceptibility to amphotericin B, itraconazole, voriconazole, posaconazole and caspofungin of Aspergillus spp. isolated from patients with haematological malignancies in Tunisia
}

Soukeina Gheith ${ }^{1,2,3}$, Fatma Saghrouni², Wadiaa Bannour ${ }^{1}$, Yosra Ben Youssef $f^{4}$, Abderrahim Khelif ${ }^{4}$, Anne-Cécile Normand ${ }^{5,6}$, Renaud Piarroux ${ }^{5,6}$, Moncef Ben Said ${ }^{2}$, Mansour Njah ${ }^{1,2}$ and Stéphane Ranque ${ }^{5,6^{*}}$

\begin{abstract}
The resistance of Aspergillus species to antifungal is increasingly reported and the knowledge of the local epidemiology and antifungal susceptibility pattern is pivotal to define adequate treatment policies. Our study aimed to: 1) describe the in vitro antifungal susceptibility profile of the Aspergillus species isolated from patients with haematological malignancies in Tunisia; 2) compare the E-test and Sensititre Yeast-One assays for the detection of paradoxical growth and trailing effect, both phenotypes commonly exhibited by Aspergillus spp. upon exposure to caspofungin and 3) to evaluate the mortality rate in patients according to the causative Aspergillus species and the antifungal treatment.

We tested amphotericin $B$, itraconazole, voriconazole, posaconazole and caspofungin against 48 Aspergillus isolates (17, A. niger; 18, A. flavus; 9, A. tubingensis; 1, A. westerdijkiae; and 1, A. ochraceus) with the E-test. Minimal inhibition concentrations were above the epidemiological cut-off values for amphotericin B in $67 \%$ of $A$. flavus strains; for caspofungin in 22\% of A. flavus strains; and for itraconazole in 22\% of A. tubingensis strains, voriconazole and posaconazole MICs were below the epidemiological cut-off values for all strains.

When exposed to caspofungin, $42 \%$ of the strains exhibited trailing effect and 38\% paradoxical growth. Trailing effect occurred in $61 \%$ of $A$. flavus strains and paradoxical growth in $62 \%$ of Aspergillus section Nigri strains. E-test and Sensititre Yeast-One assays were only fairly concordant for the detection of these phenotypes. Repeatability of both assays was high for trailing effect but poor for paradoxical growth. The relatively high frequency of amphotericin B resistant strains makes voriconazole best adapted as a first-line treatment of invasive aspergillosis from amphotericin B to voriconazole in this hospital.
\end{abstract}

Keywords: Invasive aspergillosis; Haematological malignancies; Aspergillus; In vitro susceptibility; Antifungal drugs; Amphotericin B; Itraconazole; Voriconazole; Posaconazole; Caspofungin; Paradoxical growth; Trailing effect; MIC; In vitro susceptibility testing

\footnotetext{
* Correspondence: stephane.ranque@ap-hm.fr

${ }^{5}$ Parasitology \& Mycology, CHU Timone-Adultes, Assistance

Publique-Hôpitaux de Marseille, Marseille 13005, France

${ }^{6}$ Aix-Marseille Université, IP-TPT UMR MD3, Marseille 13885, France

Full list of author information is available at the end of the article
} 


\section{Introduction}

Invasive aspergillosis (IA) is a life threatening infection, especially in neutropenic patients where it is associated with a high mortality rate (Montagna et al., 2012; Blot et al., 2012). Over the last two decades, new antifungal agents including azoles and caspofungin were developed in order to improve the prognosis of IA. In parallel, tests for Aspergillus spp. in vitro antifungal susceptibility testing were developed and commercialized (Pfaller, 2012). The extensive use of antifungal agents was associated with the emergence of azole-resistant Aspergillus spp., and caspofungin (CS) has been recommended as a salvage treatment of IA (Pfaller et al., 2008; Jarque et al., 2013). Thus, the in vitro susceptibility testing of Aspergillus spp. clinical strains to antifungal agents is required both for driving and monitoring antifungal therapy and for the global surveillance of Aspergillus spp. susceptibility (Rex and Pfaller, 2002). In vitro susceptibility tests are based on the measurement of the fungal growth in the presence of different drug concentrations so as to determine the minimum inhibitory concentration (MIC) of antifungals (Wanger, 2012). Epidemiologic cut-off values (ECVs) of the MIC were established for different Aspergillus species and different antifungal agents, in order to assess the emergence of strains with decreased susceptibility (Espinel-Ingroff et al., 2011a, Espinel-Ingroff et al. 2011b). A wild type organism being defined as a strain which does not harbor any acquired resistance to the particular antimicrobial agent being examined (Pfaller et al., 2011).

When exposed to CS, in addition to the clear end point phenotype (defined as the absence of growth at concentrations above the MIC), Aspergillus spp. exhibit two unusual in vitro testing phenotypes that are referred to as trailing effect (TE) and paradoxical growth (PG). TE is characterized by a reduced but persistent growth at concentrations above the MIC. PG is characterized by growth in the presence of low concentrations, no growth at intermediate concentrations, and growth resuming at higher concentrations (Fortwendel et al., 2010).

The aims of our study were to evaluate the in vitro antifungal susceptibility profile of Aspergillus spp. strains isolated from patients with haematological malignancies by using the E-test method, to assess both E-test ${ }^{\mathrm{Tm}}$ and Sensititre Yeast-One ${ }^{\mathrm{mm}}$ (SYO) assays for the detection of PG and TE phenotypes exhibited by Aspergillus spp. upon exposure to CS and to evaluate the mortality rate in patients according to the causative Aspergillus species and the antifungal treatment.

\section{Material and methods}

\section{Isolates}

We tested 48 clinical Aspergillus isolates, including 17 A. niger, 18 A. flavus, 9 A. tubingensis, 2 A. fumigatus, 1 $A$. westerdijkiae and $1 A$. ochraceus. These isolates were recovered from the sputa of 30 patients treated for acute leukaemia in the haematology unit of the Farhat Hached hospital of Sousse (central Tunisia). The sputa were inoculated onto Sabouraud-chloramphenicol medium and plates were incubated at 25 and $35^{\circ} \mathrm{C}$. Aspergillus sections were identified on the basis of macroscopic and microscopic characteristics of the colonies (De Hoog et al., 2000), whereas the identification at the species level was performed by using both Matrix-Assisted Laser Desorption Ionization Time-Of-Flight (MALDI-TOF) mass spectrometry on a Microflex LT ${ }^{\mathrm{TM}}$ (Bruker Daltonics, Germany) instrument (Cassagne et al., 2012) and DNA sequencing of the ITS1-5.8-ITS2 and the 28S unit D1-D2 regions of the rRNA gene, and the partial beta-tubulin (BTUB) gene (Hendrickx et al., 2012).

\section{Antifungal susceptibility testing}

We assessed the susceptibility of all the 48 isolates to the five following antifungal agents: amphotericin $B$ (AMB), itraconazole (ITR), voriconazole (VOR), posaconazole (POS) and caspofungin (CS) by using the E-test (bioMérieux, France) assay with RPMI medium (AES, France) according to the supplier's recommendations. The plates were incubated at $30^{\circ} \mathrm{C}$ for 48 hours. The MIC was determined at $100 \%$ inhibition for all tested antifungals and red at the lowest drug concentration at which the border of the elliptical inhibition intercepted the scale of the antifungal strip.

For each antifungal, we used the previously described ECVs, detailed in the Table 1, to detect the isolates within each species that might have acquired a mutational

Table 1 Epidemiological cut-off values for amphotericin, itraconazole, voriconazole, posaconazole and caspofungin according to the Aspergillus species

\begin{tabular}{|c|c|c|c|c|c|}
\hline Species & $\mathrm{AMB}^{\mathrm{a}}(\mathrm{mg} / \mathrm{l})$ & $\mathrm{ITR}^{\mathrm{b}}(\mathrm{mg} / \mathrm{l})$ & $\operatorname{VOR}^{\mathrm{c}}(\mathrm{mg} / \mathrm{l})$ & $\operatorname{POS}^{\mathrm{d}}(\mathrm{mg} / \mathrm{l})$ & $\mathrm{CS}^{\mathrm{e}}(\mathrm{mg} / \mathrm{l})$ \\
\hline A. niger & 4 & 2 & 2 & 1 & 0.25 \\
\hline A. flavus & 4 & 1 & 1 & 0.5 & 0.5 \\
\hline A. fumigatus & 4 & 1 & 1 & 0.5 & 1 \\
\hline A. tubingensis & 4 & 2 & 2 & 1 & 0.25 \\
\hline Aspergillus section Circumdati & 4 & 1 & 1 & 0.5 & 1 \\
\hline
\end{tabular}

amphotericin; ${ }^{b}$ itraconazole; ${ }^{c}$ voriconazole; ${ }^{d}$ posaconazole; ${ }^{\mathrm{e}}$ caspofungin. 
resistance mechanism to a given agent (Espinel-Ingroff et al., 2011a, Espinel-Ingroff et al. 2011b; Pfaller et al., 2011). Because ECVs had not been established for A. tubingensis (section Nigri) and for the Circumdati section, we used $A$. niger's ECVs for $A$. tubingensis and those of A. fumigatus for species of section Circumdati.

We assessed both E-test and SYO (Trek Diagnostic Systems, Ltd., United Kingdom) assays for the detection of PG and TE phenotypes exhibited by Aspergillus spp. upon exposure to CS. Therefore, we analysed the CS in vitro phenotypes of 10 randomly selected isolates (4 A. niger, 4 A. flavus and 2 A. tubingensis) for which both E-test and SYO assays were performed in triplicate. The SYO was performed according the supplier's recommendations. Briefly, the inoculum suspension was prepared from a 72 hours culture grown on Sabouraud dextrose agar and adjusted to the density of a 0.5 McFarland standard. The plates were incubated at $30^{\circ} \mathrm{C}$ and red at 24 and 48 hours by visual inspection. The MIC value corresponds to the first well that shows a change in colour from pink to purple (indicating inhibition of growth). ATCC 22019 and ATCC 6258 were used as quality control strains.

\section{Data analysis}

MICs data of the E-test assay were presented as the $\mathrm{MIC}$ range, $\mathrm{MIC}_{50}$, and $\mathrm{MIC}_{90}$ for each species. The inter-assay concordance was estimated via Cohen's kappa coefficient, by considering triplicate assays results

Table 2 MIC range, MIC50 and MIC90 of the 48 clinical Aspergillus isolates

\begin{tabular}{|c|c|c|c|c|c|}
\hline \multirow[b]{2}{*}{ Species } & \multirow[b]{2}{*}{ Antifungal agent } & \multicolumn{4}{|c|}{ MIC (mg/l) } \\
\hline & & Range & $\mathrm{MIC}_{50}$ & $\mathrm{MIC}_{90}$ & $\%>E^{\prime} V^{f}$ \\
\hline \multirow[t]{5}{*}{ A. niger $(n=17)$} & $\mathrm{AMB}^{\mathrm{a}}$ & $0.04-1.5$ & 0.44 & 0.75 & 0 \\
\hline & $\mathrm{ITR}^{\mathrm{b}}$ & $0.38-2$ & 0.98 & 2 & 0 \\
\hline & VOR $^{c}$ & $0.05-0.12$ & 0.1 & 0.13 & 0 \\
\hline & $\operatorname{POS}^{d}$ & $0.05-0.25$ & 0.12 & 0.25 & 0 \\
\hline & $\mathrm{CS}^{\mathrm{e}}$ & $0.008-0.12$ & 0.03 & 0.07 & 0 \\
\hline \multirow[t]{5}{*}{ A. flavus $(n=18)$} & AMB & $0.5-0.32$ & 6 & 20.8 & 66.6 \\
\hline & ITR & $0.25-1$ & 0.5 & 0.83 & 0 \\
\hline & VOR & $0.06-0.5$ & 0.19 & 0.25 & 0 \\
\hline & POS & $0.06-0.25$ & 0.19 & 0.25 & 0 \\
\hline & CS & $0.004-32$ & 0.064 & 32 & 22.2 \\
\hline \multirow[t]{5}{*}{ A. tubingensis $(n=9)$} & AMB & $0.09-0.5$ & 0.24 & 0.4 & 0 \\
\hline & ITR & $0.25-8$ & 0.19 & 4.8 & 22.2 \\
\hline & VOR & $0.064-0.38$ & 0.13 & 0.38 & 0 \\
\hline & POS & $0.047-0.25$ & 0.12 & 0.25 & 0 \\
\hline & CS & $0.008-0.012$ & 0.01 & 0.012 & 0 \\
\hline \multirow[t]{5}{*}{ A. fumigatus $(\mathrm{n}=2)$} & AMB & $3-6$ & $N R^{9}$ & NR & NR \\
\hline & ITR & $0.8-0.5$ & NR & NR & NR \\
\hline & VOR & $0.06-0.13$ & NR & NR & NR \\
\hline & POS & $0.06-0.13$ & NR & NR & NR \\
\hline & CS & $0.06-0.06$ & 0.064 & NR & NR \\
\hline \multirow[t]{5}{*}{ Aspergillus section Circumdati $(\mathrm{n}=2)$} & AMB & $3-24$ & NR & NR & NR \\
\hline & ITR & $0.38-1$ & NR & NR & NR \\
\hline & VOR & $0.04-0.06$ & NR & NR & NR \\
\hline & POS & $0.09-0.25$ & NR & NR & NR \\
\hline & CS & $0.023-0.13$ & NR & NR & NR \\
\hline \multirow[t]{5}{*}{ All species $(n=48)$} & AMB & $0.04-32$ & 0.63 & 13.2 & 25 \\
\hline & ITR & $0.25-8$ & 0.5 & 2 & 4.1 \\
\hline & VOR & $0.04-0.5$ & 0.13 & 0.25 & 0 \\
\hline & POS & $0.05-0.25$ & 0.13 & 0.25 & 0 \\
\hline & CS & $0.004-32$ & 0.023 & 0.125 & 8.3 \\
\hline
\end{tabular}

amphotericin; ${ }^{{ } \text {itraconazole; }}{ }^{\mathrm{c}}$ voriconazole; ${ }^{\mathrm{d}}$ posaconazole; ${ }^{\mathrm{e}}$ caspofungin; ${ }^{\mathrm{f}}$ epidemiologic cut-off value; ${ }^{\mathrm{g}}$ not relevant. 
as independent, using the following interpretation: no match $[<0]$, poor agreement $[0-0.2]$, fair agreement [0.21-0.4], moderate agreement [0.41-0.6], good agreement $[0.61-0.8]$, very good agreement [0.81-1]. Logistic regression analysis was performed to calculate the risk of a patient's fatal outcome associated with the first-line antifungal treatment. All statistical analyses were performed using SAS, version 9.2 (SAS Institute, Cary, NC).

This study was approved by the Comité d'Ethique et de Recherche de l'Hôpital Universitaire Farhat Hached de Sousse.

\section{Results}

\section{Antifungal susceptibility}

The MIC data are shown in Table 2. The $\mathrm{MIC}_{50}$ of all investigated antifungals for all Aspergillus species were low, indicating the absence of natural antifungal resistance of the tested species. When $\mathrm{MIC}_{90}$ are considered, $\mathrm{AMB}$ and ITR showed high values as compared to the remaining antifungals; thus indicating that some isolates had developed resistance to AMB and ITR.

The $\mathrm{MIC}_{50}$ and $\mathrm{MIC}_{90}$ of $\mathrm{AMB}$ were 0.63 and $13.2 \mathrm{mg} / \mathrm{l}$, respectively, for all species. The $\mathrm{MIC}_{90}$ of $\mathrm{AMB}$ was much lower for both $A$. tubingensis $(0.75 \mathrm{mg} / \mathrm{l})$ and $A$. niger $(0.4 \mathrm{mg} / \mathrm{l})$ than for A. flavus $(20.8 \mathrm{mg} / \mathrm{l})$. Twelve $(66.6 \%)$ A. flavus isolates had AMB MIC above the corresponding $\mathrm{ECV}$, indicating that these isolates have acquired a resistance to AMB. All the 36 remaining investigated strains were susceptible to AMB.

The $\mathrm{MIC}_{50}$ and $\mathrm{MIC}_{90}$ of ITR were 0.5 and $2 \mathrm{mg} / \mathrm{l}$, respectively, for all species. Only the black Aspergillus species had relatively high ITR $\mathrm{MIC}_{90}: 2 \mathrm{mg} / \mathrm{l}$ for A. niger and $4.8 \mathrm{mg} / \mathrm{l}$ for A. tubingensis. Two (22\%) A. tubingensis isolates had ITR MIC $>\mathrm{ECV}$. The MIC of CS were $>$ ECV in only $4(22 \%)$ A. flavus isolates. The MIC of VOR and POS were below the ECVs in all the 48 tested isolates.

\section{Caspofungin in vitro susceptibility testing phenotypes}

In order to estimate the frequency of the different phenotypes exhibited by Aspergillus upon in vitro CS exposure, we considered the results of the E-test susceptibility profiles. The CS in vitro testing phenotypes are illustrated in Figure 1. Out of the 48 tested Aspergillus isolates, 10 (21\%) exhibited a clear end point, 20 (42\%) TE and 18 (38\%) PG (Table 3). TE was more frequent with A. flavus (61\%) and PG was only observed with the Aspergillus section Nigri (A. niger and $A$. tubingensis) isolates (61.5\%). The mean CS concentration at which Aspergillus isolates growth resumed was $0.19 \pm 0.15 \mathrm{mg} / \mathrm{l}$.

We further compared the repeatability of the different CS phenotypes with both E-test and SYO assays and estimated the inter-assay concordance. PG never occurred simultaneously with both E-test and SYO. The TE phenotype was

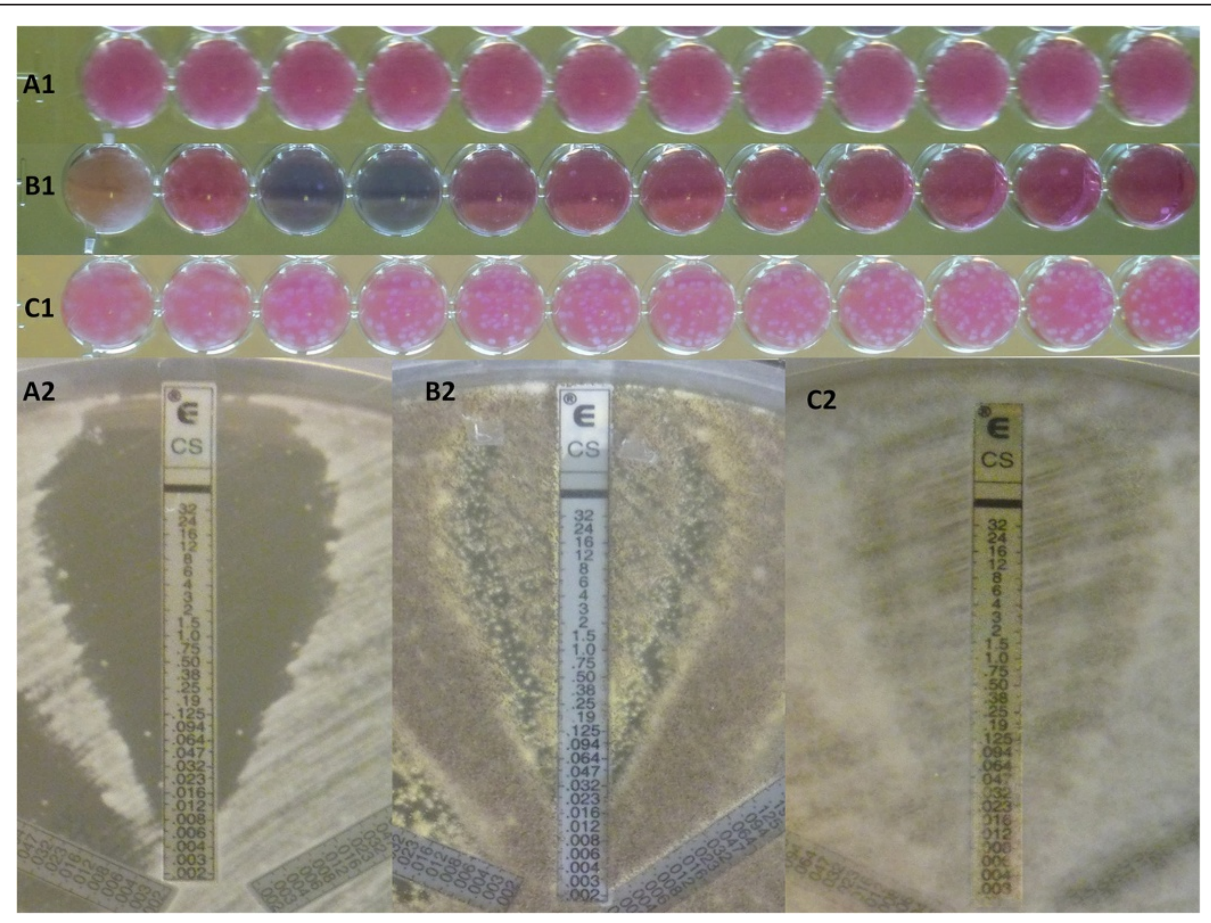

Figure 1 Caspofungin in vitro testing phenotypes of Aspergillus spp. with the Sensititre Yeast-One (SYO, Trek Diagnostic Systems) and the E-test (bioMérieux) assays. Clear end point (A. tubingensis, panel A1, SYO; panel A2, E-test), Paradoxical growth (A. niger, panel B1, SYO; panel B2, E-test) and Trailing effect (A. flavus, panel C1, SYO; panel C2, E-test). 
Table 3 Caspofungin E-test susceptibility testing phenotypes in 48 Aspergillus isolates

\begin{tabular}{|c|c|c|c|c|c|c|}
\hline \multirow[b]{2}{*}{ A. niger $(n=17)$} & \multicolumn{2}{|c|}{ Clear end point } & \multicolumn{2}{|c|}{ Trailing effect } & \multicolumn{2}{|c|}{ Paradoxical growth } \\
\hline & 1 & $(6 \%)$ & 4 & $(23.5 \%)$ & 12 & $(70.5 \%)$ \\
\hline A. tubingensis $(\mathrm{n}=9)$ & 4 & $(44.5 \%)$ & 1 & $(11 \%)$ & 4 & $(44.5 \%)$ \\
\hline A. flavus $(n=18)$ & 5 & $(28 \%)$ & 11 & $(61 \%)$ & 2 & $(11 \%)$ \\
\hline A. fumigatus $(\mathrm{n}=2)$ & 0 & & 2 & $(100 \%)$ & 0 & \\
\hline A. ochraceus $(\mathrm{n}=1)$ & 0 & & 1 & $(100 \%)$ & 0 & \\
\hline A. westerdijkiae $(n=1)$ & 0 & & 1 & $(100 \%)$ & 0 & \\
\hline All species $(n=48)$ & 10 & $(21 \%)$ & 20 & $(42 \%)$ & 18 & $(38 \%)$ \\
\hline
\end{tabular}

highly repeatable with the $A$. flavus isolates especially when E-test was used, whereas PG phenotype was poorly repeatable using both assays (Table 4). E-test and SYO assays were fairly concordant with 0.35 and 0.26 kappa values for TE and PG, respectively.

\section{Mortality rate according to the Aspergillus species and antifungal treatment}

Among the specimens obtained from the 18 patients who died, species of section Nigri were isolated from 10 (55.5\%) sputa, species of section Flavi from 6 (33.3\%) sputa and other Aspergillus species from 2 (11.1\%) sputa (Table 5). The association of Aspergillus species with a fatal outcome was not statistically significant $(\mathrm{p}=0.81)$. Among the patients with a fatal outcome, seven had been treated with $\mathrm{AMB}$, two with VOR and nine received no antifungal treatment. The first-line antifungal treatment was statistically significantly $(p=0.02)$ associated with the patients' outcome. Logistic regression analysis indicated that the risk of death was increased in the patients treated with $\mathrm{AMB}$ $(\mathrm{OR}=3.89,95 \% \mathrm{CI}[0.80$ to 18.98$], \mathrm{p}=0.0144)$ and decreased in the patients treated with VOR $(\mathrm{OR}=0.28$, 95\% CI [0.50 to 1.54$], \mathrm{p}=0.0233$ ) when compared to those who were not treated by antifungals.

\section{Discussion}

Our study revealed that Aspergillus of sections Nigri and Flavi were the most frequently isolated species from our patients' airways samples. This reflects the particular $A s$ pergillus species spectrum involved in IA in Tunisia that contrasts with the prevalent data from developed Northern countries where Aspergillus section Fumigati is responsible for more than $80 \%$ of IA cases, followed by the section Flavi (10\%); the sections Nigri, Nidulantes and Terrei causing the remaining 10\% of cases (Krishnan et al., 2009; Wald et al., 1997). This finding may be explained by the qualitative and quantitative variations in environmental Aspergillus flora according to local climate and points out the need for in vitro susceptibility testing of clinical strains in order to select the appropriate predictive therapy.

All the tested strains of sections Nigri, Fumigati and Circumdati were susceptible to AMB with $\mathrm{MIC}_{90}<\mathrm{ECV}$. This finding is in accordance with the results of other studies where no AMB resistance was reported in isolates of the section Nigri (Hadrich et al., 2012a). EspinelIngroff et al. showed that there were no MIC above the ECV for A. niger (Espinel-Ingroff et al., 2011a). Similarly low AMB MIC (MIC mostly $<0.5 \mathrm{mg} / \mathrm{l}$ ) have been recently reported by Alcazar-Fuoli et al. and Baddley et al.

Table 4 Repeatability of triplicate caspofungin E-test and SYO in vitro susceptibility testing results

\begin{tabular}{|c|c|c|c|c|c|c|c|}
\hline \multirow[t]{2}{*}{ Isolate } & \multirow[t]{2}{*}{ Species } & \multicolumn{3}{|c|}{ E-test } & \multicolumn{3}{|c|}{ SYO } \\
\hline & & Clear end point & Trailing effect & Paradoxical growth & Clear end point & Trailing effect & Paradoxical growth \\
\hline 1 & A. niger & 0 & 2 & 1 & 2 & 1 & 0 \\
\hline 2 & A. niger & 0 & 2 & 1 & 1 & 0 & 2 \\
\hline 3 & A. niger & 0 & 0 & 3 & 3 & 0 & 0 \\
\hline 4 & A. niger & 1 & 1 & 1 & 2 & 0 & 1 \\
\hline 5 & A. tubingensis & 1 & 1 & 1 & 1 & 0 & 2 \\
\hline 6 & A. tubingensis & 1 & 1 & 1 & 2 & 0 & 1 \\
\hline 7 & A. flavus & 0 & 3 & 0 & 0 & 3 & 0 \\
\hline 8 & A. flavus & 0 & 3 & 0 & 1 & 2 & 0 \\
\hline 9 & A. flavus & 0 & 3 & 0 & 0 & 3 & 0 \\
\hline 10 & A. flavus & 1 & 2 & 0 & 2 & 1 & 0 \\
\hline
\end{tabular}




\begin{tabular}{|c|c|c|c|c|}
\hline & & Death & Survival & $\mathbf{P}$ \\
\hline \multicolumn{2}{|c|}{ Aspergillus section Flavi } & $6(33 \%)$ & $12(40 \%)$ & 0.81 \\
\hline \multicolumn{2}{|c|}{ Aspergillus section Nigri } & $10(56 \%)$ & $16(53 \%)$ & \\
\hline \multicolumn{2}{|c|}{ Other Aspergillus spp. } & $2(11 \%)$ & $2(11 \%)$ & \\
\hline \multirow[t]{3}{*}{ Treatment } & AMB & $7(39 \%)$ & $3(10 \%)$ & 0.02 \\
\hline & VOR & $2(11 \%)$ & $12(40 \%)$ & \\
\hline & No & $9(50 \%)$ & $15(50 \%)$ & \\
\hline
\end{tabular}

for A. niger (Alcazar-Fuoli et al., 2009; Baddley et al., 2009).

In contrast, $66.6 \%$ of $A$. flavus isolates exceeded AMB's ECV. This finding is in line with Hadrich et al., who showed that $84 \%$ of $A$. flavus strains isolated in Sfax, a city located south of Tunisia, had a reduced susceptibility to AMB (Hadrich et al., 2012b). It is also in accordance with those reported by Lass-Flörl et al. who showed that $67 \%$ of $A$. flavus isolates in Austria were resistant to AMB and that this in vitro resistance was associated with AMB therapy failure (Lass-Flörl et al., 1998). $\mathrm{AMB}$ resistance in A. flavus was of major concern because AMB deoxycholate was the first-line treatment of IA in our hospital at the time of the study.

ITR was found to be active on all tested Aspergillus species except for $A$. niger and $A$. tubingensis. A. niger showed a reduced susceptibility to ITR. The recently described A. tubingensis species was associated with higher ITR MICs as compared to A. niger ( $4.8 \mathrm{mg} / \mathrm{l}$ vs $2 \mathrm{mg} / \mathrm{l}$ ) and MIC above ECV in $22 \%$ of isolates. The lower susceptibility of A. tubingensis strains might be related to the occurrence of a mutation, similarly to the one described by Howard et al. at position 97 in the CYP51A gene of A. awamori, another species of the section Nigri (Howard et al., 2011). The clinical impact of in vitro resistance to ITR of Aspergillus isolates is relatively modest because, especially in haematology patients, this antifungal agent is used for prophylaxis rather than for the curative treatment of IA (Döring et al., 2013).

With respect to VOR, neither resistance nor reduced susceptibility could be demonstrated in any of the strains tested. Our results contrast with those of Hendrickx et al. who reported higher MICs to both VOR and ITR in A. tubingensis (Hendrickx et al., 2012).

All strains were susceptible to POS, the MICs of which being as low as those of VOR. Our results contrast with those reported by Pfaller et al. who assessed the triazole ECVs of 1789 Aspergillus isolates and showed that the percentages of isolates for which MICs were greater than the ECVs ranged from 1.1 to $5.7 \%$ for POS, 0.0 to $1.6 \%$ for VRC and 0.7 to $4.0 \%$ for ITC (Pfaller et al., 2011).

More than $20 \%$ of $A$. flavus strains were resistant to CS. This finding contrasts with the one in all other Aspergillus species that were susceptible, with very low MICs, to CS. This relatively high resistance rate in A. flavus makes CS poorly adapted to the treatment of IA in the local hospital epidemiology setting. In contrast to our findings, AlWathiqi et al. showed that the $\mathrm{MIC}_{90}$ of A. flavus was $0.032 \mathrm{mg} / \mathrm{l}$ and MIC was above the ECV in $6 \%$ of $A$. flavus strains (Al-Wathiqi et al., 2013).

With respect to the Aspergillus species isolated from our patients' samples, the low susceptibility of a significant proportion of strains to AMB and their high susceptibility to VOR, argue for the use of VOR as the firstline treatment of IA in our hospital, especially in the haematology unit. This is in line with the international recommendations (Seyedmousavi et al., 2013; Walsh et al., 2008).

By analysing the particular CS in vitro testing phenotypes, we concluded that TE and PG are not only dependent on the Aspergillus species, but also on the strain and the assay's characteristics (Fortwendel et al., 2010). These distinct phenotypic responses to increasing CS concentrations might result from differences in the genetic background of Aspergillus species. Our study is the first to report the low level of agreement between E-test and SYO and the relatively poor reproducibility of both tests in the detection of TE or PG in Aspergillus spp. These findings argue against a simple genetic effect, and are more concordant with the hypothesis that the mechanism could be a compensatory up-regulation of the cell wall components' synthesis, in response to high CS concentrations which stimulate chitin production (Fortwendel et al., 2010). Furthermore, the clinical relevance of the capacity of fungal subpopulations to survive and proliferate at high CS concentrations is debatable. Although the clinical significance of PG in fungi exposed to CS remains unclear, we were concerned by the fact that the concentration at which Aspergillus spp. growth resumed was clearly below the expected $1 \mathrm{mg} / \mathrm{l}$ plasma concentration in treated patients. Therefore, we suggest that CS would benefit of being combined with another antifungal in order to remove the PG effect (Gellen-Dautremer et al., 2010).

When the causative Aspergillus species was considered, the case fatality rate was similar between our patients. In contrast, a significant association between the outcome of the patients and the antifungal treatment was demonstrated. This association should however be cautiously interpreted, mainly because the treatments were not randomly allocated. The fact that VOR was associated with a better outcome is in agree with the current recommendations to using VOR as the first-line treatment of IA (Walsh et al., 2008). The higher case fatality rate in the patients who were treated with $\mathrm{AMB}$ as compared to those who did not receive any antifungal treatment might result from the drug's toxicity or from a more severe form of IA disease in treated patients. Furthermore, it is well known that the 
clinical outcome does not only depend on the in vitro antifungal susceptibility profile, but also on host factors, including underlying disease, cellular and humoral immune functions and antifungal agent's pharmacokinetic/pharmacodynamic properties; all these factors are considered to play a critical role in the patients' response to the treatment (Steinbach et al., 2003).

\section{Conclusions}

Aspergillus of the section Nigri and Flavi were the most frequently involved in IA in our patients with acute leukaemia. More than $2 / 3$ of $A$. flavus isolates showed a reduced susceptibility to AMB, and $22 \%$ of $A$. tubingensis showed a reduced susceptibility to ITR. Based on these findings, we recommend VOR for the first-line treatment of IA in this haematology unit. The relatively poor reproducibility of CS in vitro testing results suggests that it cannot reliably be used to predict the patients' outcome. Further studies aiming at determining the clinical significance of both TE and PG in vitro phenotypes are warranted, mainly because when they occur, Aspergillus spp. show the capacity to grow at concentrations clearly below the expected plasma drug concentration in treated patients.

\section{Abbreviations}

SYO: Sensititre Yeast-One; MIC: Minimum inhibitory concentration; ECV: Epidemiologic cut-off values; AMB: Amphotericin B; POS: Posaconazole; VOR: Voriconazole; ITR: Itraconazole; CS: Caspofungin; TE: Trailing effect; PG: Paradoxical growth; OR: Odds-ratio.

\section{Competing interests}

The authors declare that they have no competing interests.

\section{Authors' contributions}

SG, FS, MN and SR designed this study. SG, WB and ACN performed the experiments, collected and entered the data. YBY and AK managed and diagnosed the patients. SR analysed the data. SG, FS, SR, RP and MBS drafted the paper. All authors read and approved the final manuscript.

\section{Acknowledgements}

We thank all laboratories' staff who participated in this study. This work was supported by grants from the Minister of Higher Education and Scientific Research of Tunisia.

\section{Author details}

'Service d'Hygiène Hospitalière, CHU Farhat Hached, Sousse 4000, Tunisie. ${ }^{2}$ Unité de recherche UR 04SP24, Ministère de la Santé Publique, Tunis, Tunisie. ${ }^{3}$ Laboratoire de Parasitologie -Mycologie, CHU Farhat Hached, Sousse 4000, Tunisie. ${ }^{4}$ Service d'Hématologie Clinique, CHU Farhat Hached, Sousse, Tunisie. ${ }^{5}$ Parasitology \& Mycology, CHU Timone-Adultes, Assistance Publique-Hôpitaux de Marseille, Marseille 13005, France. ${ }^{6}$ Aix-Marseille Université, IP-TPT UMR MD3, Marseille 13885, France.

Received: 2 January 2014 Accepted: 7 January 2014

Published: 10 January 2014

\section{References}

Alcazar-Fuoli L, Mellado E, Alastruey-Izquierdo A, Cuenca-Estrella M, RodriguezTudela $J L$ (2009) Species identification and antifungal susceptibility patterns of species belonging to Aspergillus section Nigri. Antimicrob Agents Chemother 53:4514-4517
Al-Wathiqi F, Ahmad S, Khan Z (2013) Molecular identification and antifungal susceptibility profile of Aspergillus flavus isolates recovered from clinical specimens in Kuwait. BMC Infect Dis 13:126

Baddley JW, Marr KA, Andes DR, Walsh TJ, Kauffman CA, Kontoyiannis DP, Ito II, Balajee SA, Pappas PG, Moser SA (2009) Patterns of susceptibility of Aspergillus isolates recovered from patients enrolled in the TransplantAssociated Infection Surveillance Network. J Clin Microbiol 47:3271-3275

Blot SI, Taccone FS, Van den Abeele A-M, Bulpa P, Meersseman W, Brusselaers N, Dimopoulos G, Paiva JA, Misset B, Rello J, Vandewoude K, Vogelaers D (2012) A clinical algorithm to diagnose invasive pulmonary aspergillosis in critically ill patients. Am J Respir Crit Care Med 186:56-64

Cassagne C, Cella A-L, Suchon P, Normand A-C, Ranque S, Piarroux R (2012) Evaluation of four pretreatment procedures for MALDI-TOF MS yeast identification in the routine clinical laboratory. Med Mycol 51:371-377

De Hoog G, Guarro J, Gené J, Figueroo M (2000) Atlas of clinical fungi, 2nd edition. Centralal Bureau Voor Schmmelcultures, Utrecht

Döring M, Blume O, Haufe S, Hartmann U, Kimmig A, Schwarze C-P, Lang P, Handgretinger R, Müller I (2013) Comparison of itraconazole, voriconazole, and posaconazole as oral antifungal prophylaxis in pediatric patients following allogeneic hematopoietic stem cell transplantation. Eur J Clin Microbiol Infect Dis (in press)

Espinel-Ingroff A, Cuenca-Estrella M, Fothergill A, Fuller J, Ghannoum M, Johnson E, Pelaez T, Pfaller MA, Turnidge J (2011a) Wild-type MIC distributions and epidemiological cutoff values for amphotericin B and Aspergillus spp. for the CLSI broth microdilution method (M38-A2 document). Antimicrob Agents Chemother 55:5150-5154

Espinel-Ingroff A, Fothergill A, Fuller J, Johnson E, Pelaez T, Turnidge J (2011b) Wild-type MIC distributions and epidemiological cutoff values for caspofungin and Aspergillus spp. for the CLSI broth microdilution method (M38-A2 document). Antimicrob Agents Chemother 55:2855-2859

Fortwendel JR, Juvvadi PR, Perfect BZ, Rogg LE, Perfect JR, Steinbach WJ (2010) Transcriptional regulation of chitin synthases by calcineurin controls paradoxical growth of Aspergillus fumigatus in response to caspofungin. Antimicrob Agents Chemother 54:1555-1563

Gellen-Dautremer J, Lanternier F, Dannaoui E, Lortholary O (2010) Antifungal combination therapy in invasive fungal infections. Rev Med Interne 31:72-81

Hadrich I, Makni F, Neji S, Abbes S, Cheikhrouhou F, Trabelsi H, Sellami H, Ayadi A (2012a) Invasive aspergillosis: resistance to antifungal drugs. Mycopathologia 174:131-141

Hadrich I, Makni F, Neji S, Cheikhrouhou F, Bellaaj H, Elloumi M, Ayadi A, Ranque $\mathrm{S}$ (2012b) Amphotericin B in vitro resistance is associated with fatal Aspergillus flavus infection. Med Mycol 51:371-377

Hendrickx M, Beguin H, Detandt M (2012) Genetic re-identification and antifungal susceptibility testing of Aspergillus section Nigri strains of the BCCM/IHEM collection. Mycoses 55:148-155

Howard SJ, Harrison E, Bowyer P, Varga J, Denning DW (2011) Cryptic species and azole resistance in the Aspergillus niger complex. Antimicrob Agents Chemother 55:4802-4809

Jarque I, Tormo M, Bello JL, Rovira M, Batlle M, Julià A, Tabares S, Rivas C, Fernández-Sevilla A, García-Boyero R, Debén G, González-Campos J, Capote FJ, Sanz MA, Spanish Pethema Group (2013) Caspofungin for the treatment of invasive fungal disease in hematological patients (ProCAS Study). Med Mycol 51:150-154

Krishnan S, Manavathu EK, Chandrasekar PH (2009) Aspergillus flavus: an emerging non-fumigatus Aspergillus species of significance. Mycoses 52:206-222

Lass-Flörl C, Kofler G, Kropshofer G, Hermans J, Kreczy A, Dierich MP, Niederwieser D (1998) In-vitro testing of susceptibility to amphotericin B is a reliable predictor of clinical outcome in invasive aspergillosis. J Antimicrob Chemother 42:497-502

Montagna MT, De Giglio O, Napoli C, Lovero G, Caggiano G, Delia M, Pastore D, Santoro N, Specchia G (2012) Invasive fungal infections in patients with hematologic malignancies (Aurora project): lights and shadows during 18-months surveillance. Int J Mol Sci 13:774-787

Pfaller MA (2012) Antifungal drug resistance: mechanisms, epidemiology, and consequences for treatment. Am J Med 125:S3-S13

Pfaller MA, Messer SA, Boyken L, Rice C, Tendolkar S, Hollis RJ, Diekema DJ (2008) In vitro survey of triazole cross-resistance among more than 700 clinical isolates of Aspergillus species. J Clin Microbiol 46:2568-2572

Pfaller M, Boyken L, Hollis R, Kroeger J, Messer S, Tendolkar S, Diekema D (2011) Use of epidemiological cutoff values to examine 9-year trends in susceptibility of Aspergillus species to the triazoles. J Clin Microbiol 49:586-590 
Rex JH, Pfaller MA (2002) Has antifungal susceptibility testing come of age? Clin Infect Dis 35:982-989

Seyedmousavi S, Mouton JW, Verweij PE, Brüggemann RJM (2013) Therapeutic drug monitoring of voriconazole and posaconazole for invasive aspergillosis. Expert Rev Anti Infect Ther 11:931-941

Steinbach WJ, Stevens DA, Denning DW (2003) Combination and sequential antifungal therapy for invasive aspergillosis: review of published in vitro and in vivo interactions and 6281 clinical cases from 1966 to 2001. Clin Infect Dis 37(Suppl 3):S188-S224

Wald A, Leisenring W, van Burik JA, Bowden RA (1997) Epidemiology of Aspergillus infections in a large cohort of patients undergoing bone marrow transplantation. J Infect Dis 175:1459-1466

Walsh TJ, Anaissie EJ, Denning DW, Herbrecht R, Kontoyiannis DP, Marr KA, Morrison VA, Segal BH, Steinbach WJ, Stevens DA, van Burik J-A, Wingard JR, Patterson TF, Infectious Diseases Society of America (2008) Treatment of aspergillosis: clinical practice guidelines of the Infectious Diseases Society of America. Clin Infect Dis 46:327-360

Wanger A (2012) Antifungal Susceptibility Testing Methods: Non-CLSI Methods for Yeast and Moulds. In: Hall GS (ed) Interactions of yeasts, moulds, and antifungal agents. Humana Press, New York, USA, pp 75-87

doi:10.1186/2193-1801-3-19

Cite this article as: Gheith et al:: In vitro susceptibility to amphotericin B, itraconazole, voriconazole, posaconazole and caspofungin of Aspergillus spp. isolated from patients with haematological malignancies in Tunisia. SpringerPlus 2014 3:19.

\section{Submit your manuscript to a SpringerOpen ${ }^{\circ}$ journal and benefit from:}

- Convenient online submission

- Rigorous peer review

- Immediate publication on acceptance

- Open access: articles freely available online

- High visibility within the field

- Retaining the copyright to your article 